\title{
Completeness and Decidability in Sequence Logic
}

\author{
Marc Bezem $^{1}$, Tore Langholm ${ }^{2}$, and Michał Walicki ${ }^{1}$ \\ 1 Department of Computer Science \\ University of Bergen \\ $\{$ bezem, michal\}@ii.uib.no \\ 2 Department of Informatics \\ University of Oslo \\ tore.langholm@ifi.uio.no
}

\begin{abstract}
Sequence logic is a parameterized logic where the formulas are sequences of formulas of some arbitrary underlying logic. The sequence formulas are interpreted in certain linearly ordered sets of models of the underlying logic. This interpretation induces an entailment relation between sequence formulas which strongly depends on which orderings one wishes to consider. Some important classes are: all linear orderings, all dense linear orderings and all (or some specific) wellorderings.

For all these classes one can ask for a sound and complete proof system for the entailment relation, as well as for its decidability. For the class of dense linear orderings and all linear orderings we give sound and complete proof systems which also yield decidability (assuming that the underlying logic is sound, complete and decidable).

The entailment relation can be expressed in the first-order theory of the ordering. Consequently, if the latter theory is decidable, then so is the corresponding entailment relation. Even if the first-order theory of every ordinal is known to be decidable, we did not find a natural proof system. We formulate some open problems on the entailment relation in the case of well-orderings.
\end{abstract}

\section{Introduction}

Sequence logic is a parameterized logic where the formulas are sequences of formulas of some underlying logic. It can be viewed as a subsystem of linear temporal logic where the temporal aspects are completely separated from other logical aspects. This separation makes it easy to change the underlying logic without having to rethink the temporal aspects. Sequence logic has recently been proposed in [11] and its virtues for modelling dynamically changing knowledge are studied in [9].

A simple example is the Muddy Children Puzzle in epistemic logic, see [5]. Here each child's knowledge is dynamically extended every round, a process which can be described by the sequence formula $a_{1} ; \ldots ; a_{n}$, where events causing changes are modeled exclusively as changes in the states expressed by the 
successive formulas. The problem is to prove that after $m$ rounds, where $m$ is the number of children, each child knows that it is muddy. In sequence logic this problem can be casted as the entailment $a_{1} ; \ldots ; a_{m} \models \top ; b$, where $T ; b$ expresses that eventually each child knows that it is muddy. The underlying logic will, most naturally, be some epistemic logic. The example is simple in that the children's knowledge increases monotonically. As a consequence, the example is valid with respect to any ordering. More interesting examples are exhibited later the sequel to discriminate between various classes of orderings: after the proof of Theorem 4, in Section 3 (p. 7) and after the proof of Lemma 12.

In this paper we give sound and complete proof systems for sequence logic and draw some conclusions on decidability of entailment. We start by reviewing some necessary preliminaries.

\section{$1.1 \quad$ Orderings}

An ordering $(D,<)$ is an irreflexive and transitive relation $<$ on a non-empty set $D$. The non-strict variant (reflexive closure) of $<$ is denoted by $\leq$. An ordering is linear if, for any $x, y \in D$, either $x<y$ or $x=y$ or $y<x$. A least (greatest) element is an $x \in D$ such that $x \leq y(y \leq x)$ for all $y \in D$. A linear ordering is right-open if it has no greatest element. An ordering is dense if for any $x, y \in D$ with $x<y$, there exists a $z \in D$ with $x<z<y$. A wellordering is a linear ordering in which every non-empty subset has a least element. In a right-open wellordering every element $x \in D$ has a successor, that is, the least element $y \in D$ with $x<y$. Any element of a wellordering that is not the least element of the ordering nor the successor of another element, is called a limit. Classes of wellorderings modulo isomorphy are called ordinals. An important ordinal is $\omega$, the class of the natural numbers equipped with their natural ordering.

Unless explicitly stated otherwise, we assume all orderings to be linear, rightopen and to have a least element, and we will denote this class by LO. The subclasses of dense orderings and of wellorderings are denoted by DLO and $\mathrm{WO}$, respectively.

Given an ordering $<$ and $d<d^{\prime}$, we use the common notation $\left[d, d^{\prime}\right)$ to denote the left-closed, right-open interval from $d$ to $d^{\prime}$, that is, the set $\{x \in D \mid d \leq x<$ $\left.d^{\prime}\right\}$. We use $[d, \infty)$ to denote the set of elements that are greater than or equal to $d$. For a function $s: D \rightarrow X$ we use $s(I)$ to denote the $s$-image of interval $I$.

\subsection{Sequence Logic}

The formulas of sequence logic are non-empty sequences $a_{1} ; \ldots ; a_{n}$ where each $a_{i}$ is a formula of some underlying logic, ul, which is a fixed parameter.

\section{Notation and Terminology 1}

1. The language of $\mathrm{ul}$ consists of a set of formulas which are denoted by the initial lower case Latin letters, $a, b, \ldots$ The formulas of sequence logic, sequence formulas, are denoted by the initial lower case Greek letters $\alpha, \beta, \ldots$ We write 
$\alpha ; \beta$ for the concatenation of sequence formulas $\alpha$ and $\beta$. Similarly for $\alpha ; a ; \beta$ and $\alpha ; a ; b ; \beta$ when $a$ and $b$ are ul formulas. $h d(\alpha)$ denotes the first formula of the sequence formula $\alpha$.

2. If $A$ is a set of $\mathrm{ul}$ formulas, $A^{l}$ denotes the set of sequences of length $l$ of formulas from $A$. For any single formula $a, a^{l}$ denotes the sequence of $l$ occurrences of $a$. For a formula $b$ and sequence formula $\alpha=a_{1} ; \ldots ; a_{n}$ we write $b \circ \alpha$ for the sequence formula $b \wedge a_{1} ; \ldots ; b \wedge a_{n}$ formed by conjoining $b$ to each member of $\alpha$ (whenever ul contains conjunction $\wedge$ ).

3. We use $=$ both for satisfaction and for entailment, both in sequence logic and in ul. To disambiguate this overloading, the types of the lhs and the rhs are crucial: $s=a$ denotes satisfaction of a in a ul structure $s ; s=\alpha$ denotes satisfaction of $\alpha$ in a structure $s$ in sequence logic. For a set of ul structures $\mathcal{S}, \mathcal{S}=a$ means $s=a$ for all $s \in \mathcal{S}$. We let $a \models b$ express entailment in $\mathrm{ul}$. In sequence logic, entailment will depend on a class of orderings $\mathcal{C}$ and this will be expressed by $\alpha \models_{\mathcal{C}} \beta$.

A structure of sequence logic is a tuple $S=\left(d_{0}, D,<, s\right)$ where $(D,<)$ is an ordering, $d_{0}$ its least element and $m$ a mapping from $D$ to models of the underlying logic. We identify the structure $S$ with the mapping $s$ when the ordering is clear from the context.

A structure $S$ satisfies a sequence formula $\alpha=a_{1} ; \ldots ; a_{n}$ if there exist $d_{1}, \ldots, d_{n-1} \in D, d_{0}<d_{1}<\cdots<d_{n-1}$, such that for all $1 \leq i<n$ we have $s\left(\left[d_{i-1}, d_{i}\right)\right) \models a_{i}$ and $s\left(\left[d_{n-1}, \infty\right)\right) \models a_{n}$. Satisfaction of $\alpha$ in $S$ will also be denoted by $s=\alpha$ if the structure $S$ is clear from the context.

The satisfaction relation defined in the previous paragraph gives rise to the following entailment relation: Given a class $\mathcal{C}$ of orderings we define $\alpha \models_{\mathcal{C}} \beta$ if for all structures $S=\left(d_{0}, D,<, s\right)$ with $\left(d_{0}, D,<\right) \in \mathcal{C}$ we have $s \models \beta$ whenever $s \models \alpha$.

The operator _; _ corresponds to the chop operator, e.g., [7,6]. Sequence logic can also be viewed as a fragment of linear-time temporal logic (LTL). For example, $a_{1} ; \ldots ; a_{n}$ can be expressed in LTL as $a_{1} \wedge\left(a_{1} U\left(a_{2} \wedge\left(a_{2} U \ldots U\left(\neg\left(\top U \neg a_{n}\right)\right) \ldots\right)\right)\right.$. Here $U$ is the until-operator and $T$ is always true. We are not aware of the separate study of this fragment elsewhere. Complexity theoretic questions are not considered in the present paper. However, it would be interesting to investigate whether the restricted expressivity of sequence logic results in lower complexity. To give a concrete example of such a question: in [8] it is proved that satisfiability of LTL with $U$ and based on $\omega$ is PSPACE-complete. This constitutes an upper bound for the complexity of $\models_{\omega}$, with co-NP as an obvious lower bound, both with classical propositional logic as ul. The interesting question is now: what is the exact computational complexity of $=_{\omega}$ ?

The main novelty and strength of sequence logic is its parameterization by the underlying logic ul. The only general assumption about the derivability relation $\vdash$ of $\mathrm{ul}$ is that it satisfies the classical closure properties, [10,2], namely ( $X, Y$ range over sets of formulas):

extension: $X \vdash a$ if $a \in X$ 
idempotence: $X \vdash a$ if $\{b \mid X \vdash b\} \vdash a$

monotonicity: $Y \vdash a$ if for some $X \subseteq Y: X \vdash a$

We will use classical propositional logic as the main example of ul, but soundness, completeness and decidability results below only use the fact the underlying logic is sound, complete (strongly, that is, $\Gamma \vdash a$ if $\Gamma \models a$ ) and decidable, respectively. Combinations with other underlying logics can be easily conceived and some examples can be found in $[11,9]$. In some cases, we assume that ul is closed under propositional connectives with the usual semantics but even then the results apply to an arbitrary logic extending the classical propositional one.

Table 1 gives some rules of inference for sequence logic based on a proof system $\vdash$ for the underlying logic. This minimal proof system, denoted $\vdash_{\text {min }}$, will be augmented with distinct rules depending on the class of orderings.

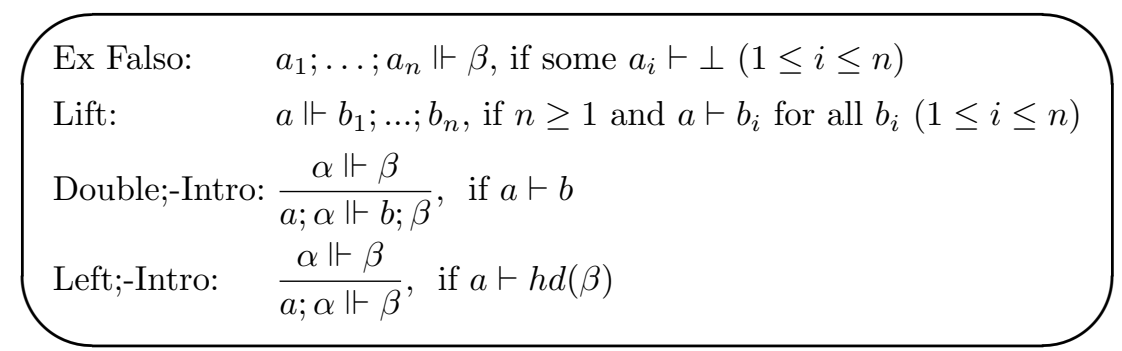

Table 1. Axioms and rules for $\Vdash_{\text {min }}$.

Theorem 2. The rules of $\vdash_{\min }$ are sound for $\models_{\mathrm{LO}}$, provided that $\vdash$ is sound for $=$ in ul.

Proof. Soundness of Ex Falso and Lift is trivial.

For Double;-Intro, assume $\alpha \models_{\text {LO }} \beta$ and $a \vdash b$ and let $s \models a ; \alpha$. Then the first interval which is used by $s$ to satisfy $a$ can also be used to satisfy $b$, relying on the soundness of the ul. We can restrict $s$ to the ordering starting with the second interval and get a model $s^{\prime}=\alpha$, so $s^{\prime} \models \beta$. It follows that $s \models b$; $\beta$, and hence $a ; \alpha \models_{\text {LO }} b ; \beta$.

For Left;-Intro, assume $\alpha \models_{\text {LO }} \beta$ and $a \vdash h d(\beta)$ and let $s \models a ; \alpha$. Then the first interval which is used by $s$ to satisfy $a$ can also be used to satisfy $h d(\beta)$, relying on the soundness of the ul. We can restrict $s$ to the ordering starting with the second interval and get a model $s^{\prime} \models \alpha$, so $s^{\prime} \models \beta$. It follows that $s=\beta$ by joining the first two intervals.

As a corollary to the proof, we have soundness of the proof system $\Vdash_{\text {min }}$ for any subclass of LO. 


\section{Dense Linear Orderings}

In this section we give a proof system extending $\Vdash_{\text {min }}$ which is sound and complete for the class of dense linear orderings. Inspection of the proof rules immediately gives the decidability of $\models_{\text {DLO }}$.

Note that joining two intervals, as done in the proof of Theorem 2, can always been done but that the dual operation, splitting an interval in two, cannot. For example, we do not have $a ; \neg a \models_{\omega} a ; a ; \neg a$ since the first interval can have length one. Consequently, the following rule Right;-Intro is not sound for all orderings, but it is sound for the class of dense orderings.

$$
\text { Right;-Intro: } \frac{\alpha \Vdash \beta}{\alpha \Vdash b ; \beta} \text { provided } h d(\alpha) \vdash b
$$

The system $\Vdash_{\text {DLO }}$ is obtained by adding the rule Right;-Intro to $\Vdash_{\text {min }}$.

Theorem 3. The proof system $\Vdash_{\mathrm{DLO}}$ is sound for $\models_{\mathrm{DLO}}$, i.e., if $\alpha \Vdash_{\mathrm{DLO}} \beta$ then $\alpha={ }_{\text {DLO }} \beta$.

Proof. In view of the proof of Theorem 2, which can be carried out with DLO instead of LO, it suffices to show that the rule Right;-Intro is sound. Assume $\alpha=_{\text {DLO }} \beta$ and $h d(\alpha) \vdash b$ and let $s \models \alpha$. Then by density the first interval which is used by $s$ to satisfy $h d(\alpha)$ can be split in two, say $\left[d_{0}, x\right)$ and $\left[x, d_{1}\right)$. We can use $\left[d_{0}, x\right)$ to satisfy $b$, since $h d(\alpha) \vdash b$. We can use $\left[x, d_{1}\right)$ to satisfy $h d(\alpha)$, and so $s$ restricted to $[x, \infty)$ satisfies $\alpha$ and hence $\beta$. It follows that $s=b ; \beta$.

The following construction will play a central role in the proof of completeness. Given two sequence formulas $\alpha, \beta$, of respective lenghts $n, m$, the structures of the underlying logic can be divided in at most $2^{n+m}$ equivalence classes, with two structures being in the same class iff they assign the same truth value to all formulas in $\alpha$ and $\beta$. By $\mathcal{S}(\alpha, \beta)$ we will denote some finite set of structures containing a member of each equivalence class. For any formula $c$ of the underlying logic we let $\llbracket c \rrbracket_{\mathcal{S}(\alpha, \beta)}=\{S \in \mathcal{S}(\alpha, \beta) \mid S \models c\}$. For every pair of ul formulas $a, b$ from $\alpha$ and $\beta$, respectively, we then have

$$
a \models b \Longleftrightarrow \llbracket a \rrbracket_{\mathcal{S}(\alpha, \beta)} \models b .
$$

Definition 2. Given a satisfiable $\alpha=a_{1} ; \ldots ; a_{n}$ and $\beta$, a dense $\mathcal{S}(\alpha, \beta)$ model of $\alpha, \mathrm{d} \mathcal{S}(\alpha, \beta)$ for short, is a structure defined on an interval $[q, q+n)$ of rationals with the usual ordering, where for every $1 \leq i \leq n$, all models from $\llbracket a_{i} \rrbracket_{\mathcal{S}(\alpha, \beta)}$ are densely distributed on $[q+i-1, q+i)$.

The existence of $\mathrm{d} \mathcal{S}(\alpha, \beta)$ 's follows, for instance, by distributing for each $1 \leq$ $i \leq n$ the models $\llbracket a_{i} \rrbracket_{\mathcal{S}(\alpha, \beta)}=\left\{V_{0}, \ldots, V_{k-1}\right\}, k>1$, in the following standard way on $[q+i-1, q+i)$. We describe the construction only for $q=0, i=1$, i.e., for $[0,1)$. Put $s\left(\frac{j}{k}\right)=V_{j}$ for all $0 \leq j<k$. Put $s\left(\frac{j}{k}+\frac{j^{\prime}}{k^{2}}\right)=V_{\left(j+j^{\prime}\right) \bmod k}$ 
for all $0 \leq j, j^{\prime}<k$. Continue in this way, in the $n$th round all intervals of length $k^{-n}$ are divided in intervals of length $k^{-n-1}$ and the models are assigned in a cyclic way. If $s\left(q^{\prime}\right)$ has not been defined in the above procedure then $s\left(q^{\prime}\right)$ can be chosen arbitrarily from $\llbracket a_{i} \rrbracket_{\mathcal{S}(\alpha, \beta)}$. Note that every dense $\mathcal{S}(\alpha, \beta)$-model of $\alpha$ is also a dense $\mathcal{S}(\alpha, \gamma)$-model of $\alpha$, for every subsequence $\gamma$ of $\beta$, assuming $\mathcal{S}(\alpha, \gamma) \subseteq \mathcal{S}(\alpha, \beta)$. Of course, $\mathrm{d} \mathcal{S}(\alpha, \beta)$ 's are not unique, but the arguments below do not depend on the particular choice of one.

Lemma 1. If ul is complete and $\mathrm{d} \mathcal{S}(\alpha, \beta) \models \beta$ then $\alpha \Vdash_{\text {DLO }} \beta$.

Proof. By induction on $|\alpha|+|\beta|$. Base case: Assume $\alpha=a, \beta=b$ and $\mathrm{d} \mathcal{S}(a, b) \mid=$ $b$. Then $\llbracket a \rrbracket_{\mathcal{S}(a, b)} \models b$, which implies $a \models b$ by $(2.1)$ and hence $a \vdash b$ by the completeness of ul. Hence $a \Vdash_{\text {DLO }} b$ by the Lift rule. For the induction step, let $|\alpha|+|\beta|>2$ and assume the lemma has been proved for all smaller cases. Let $S=\mathrm{d} \mathcal{S}(\alpha, \beta) \models \beta$. If $|\alpha|=1$, that is, $\alpha=a$ for some ul formula $a$, then every $b_{j}$ in $\beta$ is true in $\llbracket a \rrbracket_{\mathcal{S}(\alpha, \beta)}$, and hence $a \models b_{i}$, by $(2.1)$, and $a \vdash b_{i}$ by completeness of ul. We then get $a \Vdash_{\text {DLO }} \beta$ by one application of the rule Lift. The case in which $|\beta|=1$ is proved analogously, with repeated applications of the rule Left;-Intro instead of Lift. Now assume $\alpha=a_{1} ; \ldots ; a_{n}$ and $\beta=b_{1} ; \ldots ; b_{m}$ with $n, m>1$. Without loss of generality we may assume that $S$ has domain $[0, n)$. Let $[0, q)$ be the interval $S$ uses to satisfy $b_{1}$. We distinguish the following three cases.

$q<1$ Then the first interval that $S$ uses to satisfy $a_{1}$ overlaps with the first and the second interval that $S$ uses to satisfy $b_{1} ; b_{2}$. Hence we have, by induction hypothesis, $a_{1} \vdash b_{1}$ and $a_{1} \vdash b_{2}$. Consequently, $S$ is a dense $\mathcal{S}\left(\alpha, b_{2} ; \ldots ; b_{m}\right)$ model of $a_{1} ; \ldots ; a_{n}$ satisfying $b_{2} ; \ldots ; b_{m}$. By the induction hypothesis we get $a_{1} ; \ldots ; a_{n} \Vdash_{\text {DLO }} b_{2} ; \ldots ; b_{m}$ and by the rule Right;-Intro we get $\alpha \Vdash_{\text {DLO }} \beta$.

$q=1$ Then $a_{1} \vdash b_{1}$ and $S$ restricted to $[1, n)$ is a dense $\mathcal{S}\left(a_{2} ; \ldots ; a_{n}, b_{2} ; \ldots ; b_{m}\right)$ model of $a_{2} ; \ldots ; a_{n}$ satisfying $b_{2} ; \ldots ; b_{m}$. Now we get $\alpha \Vdash_{\text {DLO }} \beta$ by the induction hypothesis and an application of the rule Double;-Intro.

$q>1$ Then $a_{1} \vdash b_{1}$ and $S$ restricted to $[1, n)$ is a dense $\mathcal{S}\left(a_{2} ; \ldots ; a_{n}, \beta\right)$-model of $a_{2} ; \ldots ; a_{n}$ satisfying $\beta$. Now we get $\alpha \Vdash_{\text {DLO }} \beta$ by the induction hypothesis and an application of the rule Left;-Intro.

In all cases we have proved the conclusion of the lemma.

The completeness theorem follows directly from the above lemma.

Theorem 4. If $\alpha \models_{\mathrm{DLO}} \beta$ then $\alpha \Vdash_{\mathrm{DLO}} \beta$.

Proof. Assume $\alpha \models_{\text {DLO }} \beta$. If $\alpha$ is not satisfiable, then $\alpha \Vdash_{\text {DLO }} \beta$ by the Ex Falso rule. Otherwise, $\mathrm{d} \mathcal{S}(\alpha, \beta) \models \beta$ by assumption, and so $\alpha \Vdash_{\text {DLO }} \beta$ by Lemma 1 .

As an example, consider $a ; a \vee b ; b \not \nvdash_{\text {DLO }} a ; b ; a \vee b$. Semantically we can see that the entailment doesn't hold by distributing models of $a \wedge b$ and $a \wedge \neg b$ in a dense way on the second interval. Using the completeness theorem we get the same result from the observation that $a \vee b$ proves neither $a$ nor $b$, so that all applications of the ;-introduction rules are blocked. 
Since the base cases Ex Falso and Lift only use provability in the underlying logic and the other rules decrease the length of the sequence when applied bottom-up, we obtain the following corollary.

Corollary 1. If ul is decidable then so is $\models_{\mathrm{DLO}}$.

In particular, decidability of ul gives also decidability of $\vdash_{\text {min }}$.

\section{All Linear Orderings}

As an appetizer, showing that even with classical propositional logic as ul the relation $\models_{\text {LO }}$ is far from trivial, consider the following entailment

$$
a ; a \wedge b ; c \wedge(a \vee b) ; a \wedge b ; b \wedge c \models_{\mathrm{LO}} a ; b ; c ; c ; b .
$$

Intuitively, two consecutive $c$ 's needed to validate the conclusion can be found either in the interval for $c \wedge(a \vee b)$, provided that it is not a single point, or for $b \wedge c$. But it is far from obvious that every ordering satisfying the assumption can be chopped into intervals satisfying the conclusion.

In this section we give a sound, complete and decidable system $\Vdash_{\text {LO }}$ for $\models_{\text {LO }}$, under the assumption that $\mathrm{ul}$ is closed under boolean operators. We start by introducing a series of concepts and conventions which will be applied throughout this section.

\section{Notation and Terminology 5}

1. A convex set $A$ of natural numbers is such that $i \in A$ whenever $k \leq i \leq j$ are natural numbers such that $k, j \in A$. Any finite, non-empty convex set of natural numbers equals $\{k, \ldots, k+j\}$ for natural numbers $k, j$.

2. If $R$ is a binary relation and $A, B$ are any sets, then we write $R[A]$ for the set $\{j \mid \exists i \in A i R j\}$ and $R^{-1}[B]$ for the set $\{i \mid \exists j \in B i R j\}$.

3. An $n, m$-coupling is a relation $C \subseteq\{1, \ldots, n\} \times\{1, \ldots, m\}$ such that $-C[\{1, \ldots, n\}]=\{1, \ldots, m\}$ and $C^{-1}[\{1, \ldots, m\}]=\{1, \ldots, n\}$, and

$-i_{1} C j_{2}$ and $i_{2} C j_{1}$ never both hold when $i_{1}<i_{2}$ and $j_{1}<j_{2}$.

An n,m-coupling $C$ is said to be quasi-functional if it is functional on $\{1, \ldots, n-1\}$, i.e., if $C[\{i\}]$ is a singleton for every $i \in\{1, \ldots, n-1\}$.

4. Let $\alpha=a_{1} ; \ldots ; a_{n}$ and $\beta=b_{1} ; \ldots ; b_{m}$ be sequence formulas and let $R \subseteq$ $\{1, \ldots, n\} \times\{1, \ldots, m\}$ be a binary relation. We write $\alpha \vdash_{R} \beta$ if $a_{i} \vdash b_{j}$ for all $i, j$ such that $i R j$.

An important consequence of the definition of coupling in point 3 is that for any $n, m$ and $n, m$-coupling $C$ the images $C[A]$ and $C^{-1}[B]$ are convex sets of natural numbers whenever $A$ and $B$ are.

The characterisations below are proved by straightforward induction.

Lemma 2. Suppose all formulas of $\alpha$ are consistent. Then

(i) $\alpha \Vdash_{\mathrm{DLO}} \beta$ iff $\alpha \vdash_{C} \beta$ for $a|\alpha|,|\beta|$-coupling $C$. 
(ii) $\alpha \Vdash_{\min } \beta$ iff $\alpha \vdash_{C} \beta$ for a quasi-functional $|\alpha|,|\beta|$-coupling $C$.

Now, the inverse of Theorem 2 does not hold, i.e., $\Vdash_{\text {min }}$ is not complete for $\models_{\text {LO }}$. It is, however, complete for pairs $\alpha, \beta$ in a certain normal form which we now proceed to describe.

In the rest of this section we shall take particular interest in blocks of identical formulas occurring consecutively in a sequence formula. For this purpose we define $\equiv_{\alpha}$, for any sequence formula $\alpha=a_{1} ; \ldots ; a_{n}$, to be the smallest equivalence relation on $\{1, \ldots, n\}$ such that $i \equiv_{\alpha} i+1$ whenever $1 \leq i<n$ and $a_{i}=a_{i+1}$. The equivalence class of $i$ relative to $\equiv_{\alpha}$ is written $[i]_{\equiv_{\alpha}}$, and is always a convex set. We refer to the cardinality of $[i]_{\equiv_{\alpha}}$ as the padding of $i$. Hence the padding of $i$ is the size of the (maximal) block of consecutive, identical formulas in which $a_{i}$ occurs:

Definition 3. The $n, m$-coupling $C$ is said to be sparse if $C[\{i\}]$ is a singleton set for every $i \in\{1, \ldots, n-1\}$ with padding less than $m$.

We can now state the following Redistribution Lemma which will lead to the restricted completeness of $\Vdash_{\text {min }}$.

Lemma 3 (Redistribution). If $\alpha \vdash_{C} \beta$ for some sparse $|\alpha|,|\beta|$-coupling $C$, then also $\alpha \vdash_{C^{\prime}} \beta$ for some quasi-functional $|\alpha|,|\beta|$-coupling $C^{\prime}$.

Proof. Suppose $\alpha \vdash_{C} \beta$ for the sequence formulas $\alpha=a_{1} ; \ldots ; a_{n}$ and $\beta=$ $b_{1} ; \ldots ; b_{m}$ and the sparse $n, m$-coupling $C$. The first step is to define the (possibly partial) function $F$ on numbers $i \in\{1, \ldots, n\}$ by the following clauses.

- if $i \neq n$ and $i$ has padding less than $m$, then $F(i)$ is the unique member of $C[\{i\}]$.

- if $i$ has padding at least $m$, then $[i]_{\equiv_{\alpha}}=\{j, \ldots, j+k\}$ for some $j \leq i$ and $k \geq(m-1)$. Being the image of a convex set, $C\left[[i]_{\equiv_{\alpha}}\right]$ is also convex and hence equals $\left\{j^{\prime}, \ldots, j^{\prime}+k^{\prime}\right\}$ for some $j^{\prime}$ and $k^{\prime}$. As $1 \leq j^{\prime}$ and $j^{\prime}+k^{\prime} \leq m$, it follows that $k^{\prime} \leq(m-1) \leq k$. Now define

$$
F(j+r)=\left\{\begin{array}{l}
j^{\prime}+r \text { for } 0 \leq r \leq k^{\prime} \\
j^{\prime}+k^{\prime} \text { for } k^{\prime}<r \leq k
\end{array}\right.
$$

If $n$ itself has padding at least $m$, then $F$ is total on $\{1, \ldots, n\}$. Then let $C^{\prime}$ be $F$ itself, considered as a binary relation, i.e., let $C^{\prime}$ be the graph of $F$. If $n$ has padding less than $m$, then $F$ is defined only on $\{1, \ldots, n-1\}$. In that case let $C^{\prime}$ be the union of $F$ and $\{(n, j) \mid n C j\}$.

It is seen that $C^{\prime}\left[[i]_{\equiv_{\alpha}}\right]=C\left[[i]_{\equiv_{\alpha}}\right]$ for any $i$ with padding at least $m$, and that $C^{\prime}[\{i\}]$ and $C[\{i\}]$ are the same singleton set for $i<n$ with padding less than $m$, and it follows that $C^{\prime}$ is a quasi-functional coupling.

Since $i C^{\prime} j$ only if $i^{\prime} C j$ for some $i^{\prime} \in[i]_{\equiv_{\alpha}}$, the assumption $\alpha \vdash_{C} \beta$ directly implies $\alpha \vdash_{C^{\prime}} \beta$.

Definition 4. Let $\alpha=a_{1} ; \ldots ; a_{n}$ and $\beta=b_{1} ; \ldots ; b_{m}$ be sequence formulas. We say that $a_{i}$ is $\beta$-definite if $a_{i} \vdash b_{j}$ or $a_{i} \vdash \neg b_{j}$ for every $j \in\{1, \ldots, m\}$.

$\alpha$ is $\beta$-expanded if $a_{i}$ is $\beta$-definite for every $i \in\{1, \ldots, n-1\}$ with padding less than $m$. 
Lemma 4. If ul is complete, $\alpha \models_{\mathrm{LO}} \beta$ and $\alpha$ is $\beta$-expanded, then $\alpha \Vdash_{\min } \beta$.

Proof. By the Ex Falso rule, we may assume that each member of $\alpha$ is satisfiable. Let $D=\bigcup_{i=1}^{i=n} D_{i}$, where each $D_{i}$ is

$-\{i-1\}$ if $a_{i}$ is $\beta$-definite and $i \neq n$,

- the rationals in $[i-1, i)$ otherwise.

Now consider the structure $S=\left(d_{0}, D,<, s\right)$, where $d_{0}=0$ and $<$ is the standard ordering and $s$ is such that for each $i \in\{1, \ldots, n\}$,

$-s(i) \in \llbracket a_{i} \rrbracket_{\mathcal{S}(\alpha, \beta)}$ if $a_{i}$ is $\beta$-definite and $i \neq n$

- otherwise, $s$ distributes the members of $\llbracket a_{i} \rrbracket_{\mathcal{S}(\alpha, \beta)}$ densely over $D_{i}$ (cf. construction following Definition 2).

Since $S \models \alpha$ so, by assumption, $S \models \beta$. Define $C \subseteq\{1, \ldots, n\} \times\{1, \ldots, m\}$ to be such that $i C j$ iff $D_{i}$ intersects with the interval for $b_{j}$. $C$ is clearly an $n, m$-coupling, and the construction also guarantees that it is sparse: if $i<n$ has padding less than $m$, then by assumption $a_{i}$ is $\beta$-definite. Hence $D_{i}$ is a singleton and can only intersect with the interval for one $b_{j}$.

Finally $\alpha \vdash_{C} \beta$, i.e., $i C j$ implies $a_{i} \vdash b_{j}$. We argue for this in cases:

- If $D_{i}=\{i-1\}$ then $a_{i}$ is $\beta$-definite. Then $a_{i} \vdash b_{j}$ follows from the fact that the two have a common model.

- If $D_{i}=[i-1, i)$, and this interval intersects with the interval for $b_{j}$, then $b_{j}$ is true in all members of $\llbracket a_{i} \rrbracket_{\mathcal{S}(\alpha, \beta)}$. Hence $a_{i} \models b_{j}$ by $(1)$ and $a_{i} \vdash b_{j}$ by completeness of ul.

$\alpha \Vdash_{\text {min }} \beta$ follows now by the Redistribution Lemma 3 and Lemma 2.

The above Lemma is the restricted completeness referred to previously: when $\alpha$ is $\beta$-expanded then the pair $\alpha, \beta$ is in a normal form for which $\models_{\mathrm{LO}}$ and $\Vdash_{\text {min }}$ coincide. To obtain a general procedure for proving (and, in fact, deciding) whether $\alpha=_{\text {LO }} \beta$ holds, we show how to compute, given the pair $(\alpha, \beta)$ a finite set $\left\{\left(\rho_{1}, \rho_{1}^{\prime}\right), \ldots,\left(\rho_{k}, \rho_{k}^{\prime}\right)\right\}$ of pairs in normal form, such that $\alpha \models_{\text {LO }} \beta$ iff $\rho_{i} \models_{\text {LO }} \rho_{i}^{\prime}$, and hence $\rho_{i} \Vdash_{\min } \rho_{i}^{\prime}$, for every $i$. For this purpose, we introduce the following Definition.

Definition 5. The proof system $\Vdash_{\mathrm{LO}}$ is obtained by adding the following Cut rule to $\Vdash_{\text {min }}$ :

$$
\text { Cut } \frac{\alpha_{1} ; a ; a ; \alpha_{2} \Vdash \beta \quad \alpha_{1} ; a \wedge c ; \alpha_{2} \Vdash \beta \quad \alpha_{1} ; a \wedge \neg c ; \alpha_{2} \Vdash \beta}{\alpha_{1} ; a ; \alpha_{2} \Vdash \beta}
$$

In an application of the Cut rule, the formula occurrence displayed as a is referred to as the expansion formula.

In the view of Theorem 2, the following lemma is established by an easy verification of soundness of the Cut rule. 
Lemma 5. $\Vdash_{\mathrm{LO}}$ is sound for $\models_{\mathrm{LO}}$.

To show completeness (and decidability) of $\Vdash_{\text {LO }}$, we first consider the following generalizations of Cut.

Definition 6. For any sequence formula $\delta=d_{1} ; \ldots ; d_{l}$ let vals $(\delta)$ be the set of all conjunctions $c_{1} \wedge \ldots \wedge c_{l}$, where each $c_{j}$ is $d_{j}$ or $\neg d_{j}$. Now let the $\mathbf{i}-$, ii- and iii-Cut rules be the following where $l \geq 1$ (recall notational conventions $a^{l}$ and $a \circ \rho$ from Notation and Terminology 1):

$$
\begin{array}{cc}
\text { i-Cut } & \frac{\alpha_{1} ; a ; a ; \alpha_{2} \Vdash \beta \quad \alpha_{1} ; a \wedge \rho ; \alpha_{2} \Vdash \beta \text { for all } \rho \in \operatorname{vals}(\delta)}{\alpha_{1} ; a ; \alpha_{2} \Vdash \beta} \\
\text { ii-Cut } & \frac{\alpha_{1} ; a^{l+1} ; \alpha_{2} \Vdash \beta \quad \alpha_{1} ; a \circ \rho ; \alpha_{2} \Vdash \beta \text { for all } \rho \in \operatorname{vals}(\delta)^{l}}{\alpha_{1} ; a^{l} ; \alpha_{2} \Vdash \beta} \\
\text { iii-Cut } & \frac{\alpha_{1} ; a^{l+1} ; \alpha_{2} \Vdash \beta \quad \alpha_{1} ; a \circ \rho ; \alpha_{2} \Vdash \beta \text { for all } \rho \in \operatorname{vals}(\delta) \leq l}{\alpha_{1} ; a ; \alpha_{2} \Vdash \beta}
\end{array}
$$

The four cut rules are closely related. Cut is the special case of i-Cut corresponding to $\delta$ being a single ul formula $c$, while i-Cut is the special case of ii-Cut, as well as of iii-Cut, corresponding to $l=1$. The following Lemma is easy to verify and is stated without a proof.

Lemma 6. All four cut rules are sound and invertible (i.e., sound when applied bottom-up) for $\models_{\mathrm{LO}}$.

Corollary 2. If ul is decidable then so is $\models_{\mathrm{LO}}$.

Proof. For any candidate entailment $a_{1} ; \ldots ; a_{n} \models_{L O} \beta$ apply iii-Cut bottom-up, with the first $a_{i}$ which is not $\beta$-definite as expansion formula and with $\delta=\beta$ and $l=|\beta|$, to obtain $1+2^{l}+2^{2 l}+\ldots+2^{l^{2}}<2^{(l+1)^{2}}$ new items, which by Lemma 6 are all valid iff the original item was valid. Then proceed, for each of the new items, with a new bottom-up application of iii-Cut, this time using the next $a_{i^{\prime}}$ which is not $\beta$-definite as the expansion formula, etc., to obtain eventually less than $2^{n(l+1)^{2}}$ items in normal form which are all valid iff the original item was. Validity of each of these items is decidable, provided that ul is, by Corollary 1 .

From this proof we see that the system obtained by adding iii-Cut to $\Vdash_{\text {min }}$ is complete and decidable with respect to $\models_{\text {LO }}$. However, already $\Vdash_{\text {LO }}$, i.e., $\Vdash_{\text {min }}$ extended with Cut, has these properties and the rest of this Section is devoted to proving this fact by showing admissibility of iii-Cut in $\Vdash_{\text {LO }}$. The proof proceeds stepwise by showing first admissibility of i-Cut and then of ii-Cut. First, we need the following auxiliary result.

Lemma 7. If $c \vdash a$ and $\alpha_{1} ; a ; \alpha_{2} \Vdash_{\mathrm{LO}} \beta$, then $\alpha_{1} ; c ; \alpha_{2} \Vdash_{\mathrm{LO}} \beta$.

Proof. Proceeding by induction on proofs, we skip the trivial cases of the rules of $\Vdash_{\text {min }}$ and consider only the final step being an application of the Cut rule. This gives three cases to consider, corresponding to whether the $a$ mentioned 
in the lemma is the expansion formula itself, or it occurs to its left or to its right. In the two latter cases, the induction hypothesis is applied once to each of the three premises, always strengthening $a$ to $c$, while in the former case the induction hypothesis is applied twice to the first premise, strengthening $a$ to $c$, and once to each of the other premises, strengthening $a \wedge b$ and $a \wedge \neg b$ to $c \wedge b$ and $c \wedge \neg b$ respectively.

Lemma 8. i-Cut is admissible in $\Vdash_{\mathrm{LO}}$.

Proof. We prove this by induction on the length of $\delta$, which is always positive. The base case is just Cut itself; now suppose the result holds for $\delta$, and that

(1) $\alpha_{1} ; a ; a ; \alpha_{2} \Vdash_{\text {LO }} \beta$, and

(2) $\alpha_{1} ; a \wedge \rho ; \alpha_{2} \Vdash_{\text {LO }} \beta$ for all $\rho$ in $\operatorname{vals}(\delta ; b)$.

Now let $\kappa$ be an arbitrary member of vals $(\delta)$, then from (1) we obtain $\alpha_{1} ; a \wedge$ $\kappa ; a \wedge \kappa ; \alpha_{2} \Vdash_{\text {LO }} \beta$ by Lemma 7, and from (2) $\alpha_{1} ; a \wedge \kappa \wedge b ; \alpha_{2} \Vdash_{\text {LO }} \beta$ and $\alpha_{1} ; a \wedge \kappa \wedge$ $\neg b ; \alpha_{2} \Vdash_{\text {LO }} \beta$ by definition. Hence by Cut we also obtain (3) $\alpha_{1} ; a \wedge \kappa ; \alpha_{2} \Vdash_{\text {LO }} \beta$. Since $\kappa$ was arbitrary, we can now apply the induction hypothesis to (1) and (3), to obtain $\alpha_{1} ; a ; \alpha_{2} \Vdash_{\text {LO }} \beta$.

Lemma 9. ii-Cut is admissible in $\Vdash_{\mathrm{LO}}$.

Proof. We prove this by induction on $l$. The base case, for $l=1$, is just an instance of i-Cut and was shown in the previous Lemma 8. Now suppose the result holds for $l \geq 1$, and that

(1) $\alpha_{1} ; a^{l+2} ; \alpha_{2} \Vdash_{\text {LO }} \beta$, and

(2) $\alpha_{1} ; a \circ \rho ; \alpha_{2} \Vdash_{\text {LO }} \beta$ for all $\rho \in \operatorname{vals}(\delta)^{l+1}$.

For an arbitrary $\kappa \in \operatorname{vals}(\delta)^{l}$ we obtain from (1) $\alpha_{1} ; a ; a ; a \circ \kappa ; \alpha_{2} \Vdash_{\text {LO }} \beta$ by Lemma 7, and from (2) $\alpha_{1} ; a \wedge \rho ; a \circ \kappa ; \alpha_{2} \Vdash_{\text {LO }} \beta$ for all $\rho \in$ vals $(\delta)$ by definition. Hence we also obtain (3) $\alpha_{1} ; a ; a \circ \kappa ; \alpha_{2} \Vdash_{\text {LO }} \beta$ by i-Cut. Since $\kappa$ was arbitrary, we are now in a position to apply the induction hypothesis to (1) and (3), treating the first occurrence of $a$ in all the involved items as "passive", to obtain $\alpha_{1} ; a^{l+1} ; \alpha_{2} \Vdash_{\mathrm{LO}} \beta$.

Lemma 10. iii-Cut is admissible in $\Vdash_{\mathrm{LO}}$.

Proof. We prove this by induction on $l$. The base case $(l=1)$ is an instance of i-Cut shown in Lemma 8. So suppose the result holds for $l \geq 1$, and that

(1) $\alpha_{1} ; a^{l+2} ; \alpha_{2} \Vdash_{\text {LO }} \beta$, and

(2) $\alpha_{1} ; a \circ \rho ; \alpha_{2} \Vdash_{\text {LO }} \beta$ for all $\rho$ in vals $(\tau) \leq l+1$.

In particular $\alpha_{1} ; a \circ \rho ; \alpha_{2} \Vdash_{\text {LO }} \beta$ then holds for all $\rho$ in vals $(\tau)^{l+1}$, hence $\alpha_{1} ; a^{l+1} ; \alpha_{2} \Vdash_{\text {LO }}$ $\beta$ by ii-Cut. Combining this with "the rest of (2)" we then obtain $\alpha_{1} ; a ; \alpha_{2} \Vdash_{\text {LO }} \beta$ by the induction hypothesis.

Theorem 6. $\Vdash_{\mathrm{LO}}$ is sound and complete for $\models_{\mathrm{LO}}$.

The proof is immediate from the previous results. From the proofs it can also be seen that $\Vdash_{\text {LO }}$ remains complete when the use of Cut is restricted to cases in which the cut formula is chosen from formulas occurring in $\beta$. 


\section{Wellorderings}

For reasons of simplicity we focus in this section on classical propositional logic as ul. Recall that $\alpha \models_{\lambda} \beta$ denotes entailment with respect to structures $(0, \lambda,<, s)$ with $<$ the ordering on limit ordinal $\lambda$ and $s$ mapping ordinals $<\lambda$ to valuations. Using the standard translation of modal logic into first-order logic (see for example [1]) one can express the entailment relation in the first-order theory of the ordering. It is known that the first-order theory of every ordinal is decidable, see for example [3]. As a consequence, every entailment relation $\alpha \models_{\lambda} \beta$ is decidable.

The decidability of $\alpha \models_{\omega} \beta$ also follows from the (much stronger) result from [8] that $\omega$-based linear-time temporal logic is PSPACE-complete. This complexity theoretic result is extended to all countable ordinals in [4]. In the next section we give a simple argument for decidability of $\models_{\omega}$ based on a form of finite model property, Lemma 11, which may be of independent interest. In the concluding Section 4.2, we discuss some results concerning the entailment relation in the case of wellorderings and list some open problems.

\subsection{Decidability of $\models_{\omega}$}

The first step in our decidability proof is a simplification of the definition of $\models_{\omega}$. For this we use models $s$ defined on finite initial segments of $\omega$, the only place in this paper where we use orderings with a greatest element.

Definition 7. For $\alpha=a_{1} ; \ldots ; a_{n}$ and $\beta=b_{1} ; \ldots ; b_{m}$ we define

$$
k(\alpha, \beta)= \begin{cases}m & \text { if } a_{n} \models b_{1} \wedge \cdots \wedge b_{m} \\ m-j & \text { if } a_{n} \not b_{j} \text { and } a_{n} \models b_{j+1} \wedge \cdots \wedge b_{m}\end{cases}
$$

In words, the function $k$ computes the maximal length of a suffix of $\beta$ which is entailed by the last formula of $\alpha .^{3}$

Lemma 11. Let $\alpha=a_{1} ; \ldots ; a_{n}$ be satisfiable and let $\beta=b_{1} ; \ldots ; b_{m}$. Then we have $\alpha \models \omega \beta$ if and only if $a_{n} \models b_{m}$ and $s \models \beta$ for every $s$ defined on an initial segment of $\omega$ and satisfying $s=\alpha$ where the last interval (used to satisfy $a_{n}$ ) is of length $k(\alpha, \beta)$.

Proof. Let $\alpha, \beta$ be as above. In the equivalence we have to prove, the implication from right to left is the easiest. Assume the rhs and let $s=\alpha$ with $s$ defined on $\omega$. The last interval of $s$ is infinite, let $s^{-}$be $s$ with the last interval cut down to length $k(\alpha, \beta)$. Then $s^{-}$satisfies the condition of the rhs and hence $s^{-}=\beta$. By $a_{n}=b_{m}$ it follows that $s=\beta$.

For the converse, assume $\alpha \models{ }_{\omega} \beta$. Then in particular $a_{n} \models b_{m}$. Let $s \models \alpha$ be as assumed in the rhs, that is, defined on $[0, \ldots, i+k-1) \subseteq \omega$ and with the last interval $[i, i+k)$ of length $k=k(\alpha, \beta)$. In proving $s \models \beta$ we distinguish two cases.

\footnotetext{
${ }^{3}$ In this Subsection, we use propositional conjunction, as in $a_{n} \models b_{1} \wedge \ldots \wedge b_{m}$, only as an abbreviation for $a_{n} \models b_{1}$ and $\ldots$ and $a_{n} \models b_{m}$. That is, the ul need not contain propositional logic.
} 
$k=m$ Then $a_{n} \models b_{1} \wedge \cdots \wedge b_{m}$. Let $s^{+}$be $s$ extended with $\omega$ (arbitrary) models of $a_{n}$. Then $s^{+} \models \alpha$, so by the lhs we get $s^{+} \models \beta$. Since $a_{n} \models b_{1} \wedge \cdots \wedge b_{m}$ we can shift intervals that (possibly) occur to the right of $i$ to the left and shorten them to length 1 . In this way they all fit within the last interval of $s$. It follows that $s=\beta$.

$k<m$ Then $a_{n} \not \models b_{j}$ with $j=m-k$ and $a_{n} \mid=b_{j+1} \wedge \cdots \wedge b_{m}$. In the argument we will use a propositional model $V$ satisfying $a_{n} \wedge \neg b_{j}$. Let $s^{\prime}$ be $s$ with the last interval replaced by $\omega$ copies of $V$. Then we still have $s^{\prime} \models \alpha$, so by the lhs we get $s^{\prime} \models \beta$. Since $b_{j}$ is false in $V$, the $j$ th interval of $s^{\prime}$ must be to the left of $i$. Intervals used to satisfy the remaining formulas $b_{j+1}, \ldots, b_{m}$ and (possibly) occurring to the right of $i$ can be shortened and shifted to the left as in the previous case. With this new interval structure we still have $s^{\prime} \models_{\omega} \beta$. Restoring the last interval of $s$, that is, replacing the $\omega$ copies of $V$ by the models in the last interval of $s$, we get $s=\beta$.

The last step in the last case relies on $a_{n}=b_{j+1} \wedge \cdots \wedge b_{m}$.

Now that we have expressed $\models_{\omega}$ in terms of finite sequences of models we use the fact these can be viewed as words over an alphabet, where the symbols are valuations. Model classes then become languages. Let $\alpha=a_{1} ; \ldots ; a_{n}$ and $\beta=b_{1} ; \ldots ; b_{m}$ and $k=k(\alpha, \beta)$. Let $V_{1}, \ldots, V_{p}$ be all possible valuations of the atoms occurring in $\alpha, \beta$. For any proposition $a$, define $L(a)=\left\{V_{i}\left|V_{i}\right|=\right.$ $a\}$. Being a finite language consisting of one-letter words, $L(a)$ is regular. The finite models $m \models \alpha$ correspond one-to-one to words in the regular language $L(\alpha)=L\left(a_{1}\right)^{+} \ldots L\left(a_{n}\right)^{+}$, where juxtaposition stands for concatenation and + for one or more iterations (Kleene ${ }^{+}$). The finite models $s \models \alpha$ with last interval of length $k$ correspond one-to-one to words in the regular language $L(\alpha, k)=L\left(a_{1}\right)^{+} \cdots L\left(a_{n-1}\right)^{+} L\left(a_{n}\right)^{k}$. In this way we can rephrase the rhs of Lemma 11 as: $a_{n} \models b_{m}$ and $L(\alpha, k) \subseteq L(\beta)$. Since inclusion between regular languages is decidable we get the following result.

Theorem 7. If ul is decidable then so is the entailment relation $\models_{\omega}$.

Proof. We have $\alpha \models_{\omega} \beta$ if and only if either $\alpha$ is unsatisfiable, or $k(\alpha, \beta)>0$ and $L(\alpha, k(\alpha, \beta)) \subseteq L(\beta)$. All ingredients of the rhs are computable/decidable.

As an example, consider $a ; a \vee b ; b \models_{\omega} a ; b ; a \vee b$. Semantically, given $m \models a ; a \vee b ; b$ we can see this by looking at the second interval of $m$. If all models in this interval satisfy $a$ we are done. Otherwise, use the first model in the second interval that satisfies $b$ as second interval (of length 1 ) for $a ; b ; a \vee b$. The more general method would be to apply the above theorem. We can actually take two-bit sequences as symbols representing valuations: 11 represents the valuation which makes both $a$ and $b$ true, 10 makes only $a$ true, 01 only $b$, and 00 neither $a$ nor $b$. Then $L(a)$ is the regular language $\{10,11\}, L(b)$ is $\{01,11\}$ and $L(a \vee b)$ is $\{01,10,11\}$. Obviously, $k(a ; a \vee b ; b, a ; b ; a \vee b)=2$. So by Lemma 11 we have $a ; a \vee b ; b \models_{\omega} a ; b ; a \vee b$ if and only if $L(a)^{+} L(a \vee b)^{+} L(b)^{2} \subseteq L(a)^{+} L(b)^{+} L(a \vee b)^{+}$. The latter can be verified by a decision procedure for inclusion between regular languages. 


\subsection{Wellorderings and Open Problems}

The following lemma states that $=_{\lambda}$ is weakly decreasing in $\lambda$.

Lemma 12. For limit ordinals $\lambda<\lambda^{\prime}:$ if $\alpha \models_{\lambda^{\prime}} \beta$ then $\alpha=_{\lambda} \beta$.

Proof. Let $\alpha=a_{1} ; \ldots ; a_{n}, \beta=b_{1} ; \ldots ; b_{m}$ and $k=k(\alpha, \beta)$. Let $\lambda<\lambda^{\prime}$ be limit ordinals and assume $\alpha \models_{\lambda^{\prime}} \beta$. Let $s \models \alpha$ for some $\lambda$ model $s$. Let $V$ be a model of $a_{n}$ that, in case $k<m$, also satisfies $\neg b_{m-k}$. Let $[o, \lambda)$ be the last interval used by $s$ to satisfy $a_{n}$. Define $s_{o}^{V}\left(o^{\prime}\right)=s\left(o^{\prime}\right)$ if $o^{\prime}<o$ and $s_{o}^{V}\left(o^{\prime}\right)=V$ for all $o \leq o^{\prime}<\lambda^{\prime}$. In other words, $s_{o}^{V}$ is $s$ with the last interval replaced by sufficiently many copies of $V$ in order to be a $\lambda^{\prime}$ model of $\alpha$. As a consequence, $s_{o}^{V} \models \beta$. By the particular choice of $V$ we have $V \mid=b_{m-k+1} \wedge \cdots \wedge b_{m}$. Since $\lambda>o$ is a limit ordinal we have $o+\omega \leq \lambda$. Consequently, like in the proof of Lemma 11, we can shorten and shift to the left , i.e., into the interval $[o, \lambda)$, those of the last $k$ intervals that occur in $s_{o}^{V}$ to the right of $o$, obtaining a $\lambda$ model which still satisfies $\beta$. But since $a_{n} \models b_{m-k+1} \wedge \cdots \wedge b_{m}$, restoring now back the original $[o, \lambda)$ interval from $s$, we obtain that $s \models \beta$.

The implication cannot be reversed: we have $a ; a \wedge \neg b ; a ; a \wedge b \models_{\omega} a ; a \wedge \neg b ; a \wedge b$ (look at the third interval satisfying $a$ !), but not $a ; a \wedge \neg b ; a ; a \wedge b=_{\omega+\omega} a ; a \wedge$ $\neg b ; a \wedge b$. A counterexample to the latter is $(1110)^{\omega}(11)^{\omega}$, where we use the same representation of valuations by two-bit sequences as in the previous section. A trivial corollary of the previous theorem is: $\alpha \models_{\omega} \beta$ if and only if there exists $\lambda \in W O$ such that $\alpha \models_{\lambda} \beta$.

Since the wellorderings form a class, and $\models_{\omega}$ is a set, it can be expected that $\models_{\lambda}$ in Lemma 12 stabilizes. This can be made precise by the following argument. Assume by contradiction that for all $\lambda$ there exists a $\lambda^{\prime}>\lambda$ such that $\models_{\lambda^{\prime}} \subset \models_{\lambda}$. Define a function $f$ from ordinals to limit ordinals by $f(0)=\omega, f(o+1)=$ the smallest $\lambda$ such that $\models_{\lambda} \subset \models_{f(o)}$, and in the limit case $f(\lambda)=$ the smallest $\lambda^{\prime}$ such that $\models_{\lambda^{\prime}} \subset \models_{\lambda^{\prime \prime}}$, where $\lambda^{\prime \prime}$ is the supremum of all $f(o), o<\lambda$. Then we have that $o \mapsto \models_{f(o)}$ is a strictly decreasing mapping from the class of ordinals into the power set of $\models_{\omega}$, which is impossible. Surprisingly, it follows from known results (see [3]) that $\models_{\lambda}$ stabilizes in some $\lambda \leq \omega^{\omega}$ and then coincides with $\models_{\text {wo }}$. We finish by formulating some open problems.

Open Problem 8 For which $\lambda<\lambda^{\prime}<\omega^{\omega} d o \models_{\lambda}$ and $\left.\right|_{\lambda^{\prime}}$ coincide? Even the case $\lambda=\omega * 2, \lambda^{\prime}=\omega * 3$ is open.

Open Problem 9 Are there natural sound and complete proof systems for $\models_{\lambda}$ with $\lambda \geq \omega$ ?

\section{Acknowledgement}

We are indebted to Stéphane Demri for pointing out the relevance of [3] to us. 


\section{References}

1. P. Blackburn, M. de Rijke, and Y. Venema. Modal Logic. Cambridge University Press, 2001.

2. D. J. Brown and R. Suszko. Abstract logics. Dissertationes Mathematicae, 102:942, 1973

3. J.R. Büchi and D. Siefkes. The Monadic Second-Order Theory of All Countable Ordinals. Number 328 in Lecture Notes in Mathematics. Springer-Verlag, 1973.

4. S. Demri and A. Rabinovich. The complexity of temporal logic with until and since over ordinals. Submitted to LPAR'07.

5. R. Fagin, J. Halpern, Y. Moses, and M. Vardi. Reasoning about Knowledge. The MIT Press, 1995.

6. V. Goranko, A. Montanari, and G. Sciavicco. Propositional interval neighborhood temporal logics. Journal of Universal Computer Science, 9(9):1137-1167, 2003.

7. J. Halpern and Y. Shoham. A propositional modal logic of time intervals. Journal of the ACM, 38(4):935-962, 1991.

8. A. P. Sistla and E. M. Clarke. The complexity of propositional linear temporal logics. Journal of the ACM, 32(3):733-749, 1985.

9. W. Szajnkenig. Sequence Logic. PhD thesis, Department of Informatics, University of Bergen, 2007.

10. A. Tarski. Logic, Semantics, Metamathematics. Oxford University Press, 1956.

11. M. Walicki, M.A. Bezem, and W. Szajnkenig. A strongly complete logic of dense time intervals. In N. Alechina T. Ågotnes, editor, Proceedings of the Workshop on Logics for Resource- Bounded Agents, Malaga, Spain, 2006. 